\title{
Ineffective communication equals no communication: a case report of splenic hyperfunction combined with pseudothrombocytopenia
}

\author{
Yu Zhu ${ }^{1 \#}$, Yan Pan $^{2 \#}$, Pan-Fei Hou ${ }^{2}$, Li-Jing $Z_{h u^{2}}$ \\ ${ }^{1}$ Department of anaesthesiology, Huai'an Second People's Hospital and The Affiliated Huai'an Hospital of Xuzhou Medical University, Huai'an, \\ China; ${ }^{2}$ Department of Clinical Laboratory, Lianshui People's Hospital Affiliated to kangda college of Nanjing Medical University, Lianshui, China \\ \#These authors contributed equally to this work. \\ Correspondence to: Li-Jing Zhu. Lianshui County People's Hospital, No 6, Hongri Road, Lianshui 223400, China. \\ Email: zhulijing830710@126.com.
}

\begin{abstract}
Thrombocytopenia may be caused by diseases of various organ systems, especially the blood system. Certain drugs may also cause thrombocytopenia. In addition, it can result from various causes of pseudo-reduction. Therefore, a correct understanding of the causes of thrombocytopenia and their underlying mechanisms has important significance for clinical diagnosis and treatment. This study aimed to report a case of a 68-year-old woman with left upper abdominal mass and loss of appetite. The auxiliary examination showed splenomegaly and thrombocytopenia. The clinician planned to perform splenectomy. However, the laboratory physician considered that thrombocytopenia might be ethylenediaminetetraacetic acid-dependent pseudothrombocytopenia (EDTA-PTCP). After effective communication between laboratory physicians and clinicians, the patient was diagnosed with splenic hyperfunction and pseudothrombocytopenia, and finally saved from undergoing splenectomy. The patient has a good prognosis after oral medication. Thrombocytopenia in this patient is caused by both hypersplenism and EDTA antagonism which is different from a single factor in other reports. The diagnosis of hematological abnormality may be challenging for physicians, especially thrombocytopenia. Therefore, medical staff should possess a rigorous working style and a high sense of responsibility besides maintaining high professional quality. Further, they should actively, timely, and effectively communicate with auxiliary departments to avoid misdiagnosis and missed diagnosis.
\end{abstract}

Keywords: Clinical communication; pseudothrombocytopenia; splenic hyperfunction; case report

Submitted Sep 09, 2019. Accepted for publication Jun 29, 2020.

doi: 10.21037/apm-19-295

View this article at: http://dx.doi.org/10.21037/apm-19-295

\section{Introduction}

Thrombocytopenia is a common hematological problem. It is defined as a platelet count of $<100 \times 10^{9} / \mathrm{L}$, and is considered to be severe when the platelet count is $<50 \times 10^{9} / \mathrm{L}$. The underlying mechanisms of thrombocytopenia include marrow underproduction, drug-induced pregnancy, rheumatology, and pseudothrombocytopenia. If the reason for thrombocytopenia is not clear, it can lead to missed diagnosis and misdiagnosis, and even endanger the lives of patients, and cause medical disputes. This study aimed to report a case of a 68 -year-old woman with splenic hyperfunction and thrombocytopenia in The Lianshui County People's Hospital, China. Thrombocytopenia was mostly reported by a single factor in previous study $(1,2)$. In this case, thrombocytopenia was caused by both the hypersplenism of organic lesions and the hidden false reduction influenced by EDTA, which was rare in clinical treatment. In addition, it shows the importance of communication. The patient was saved from undergoing splenectomy after effective communication between clinicians and laboratory physicians. We present the following case in accordance with the CARE Reporting Checklist(3). 


\section{Case presentation}

A 68-year-old female with left upper abdominal mass for the past 6 years and loss of appetite for half a month, was admitted to The Lianshui County People's Hospital, China, on May 11, 2019. The left upper abdominal mass was not

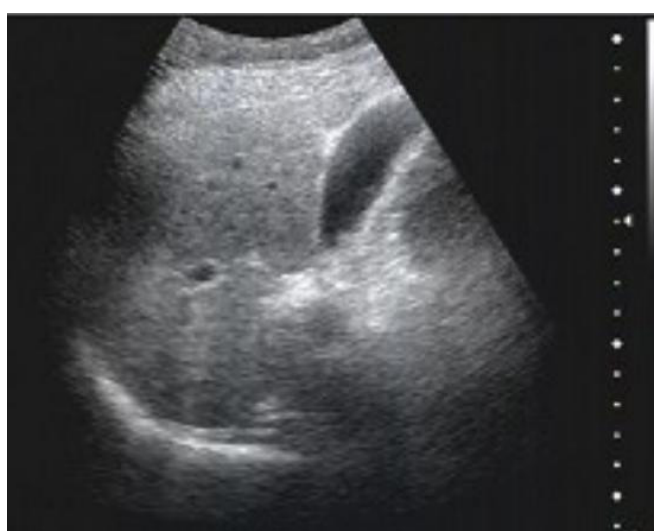

Figure 1 Abdominal ultrasound: chronic liver disease, splenomegaly.

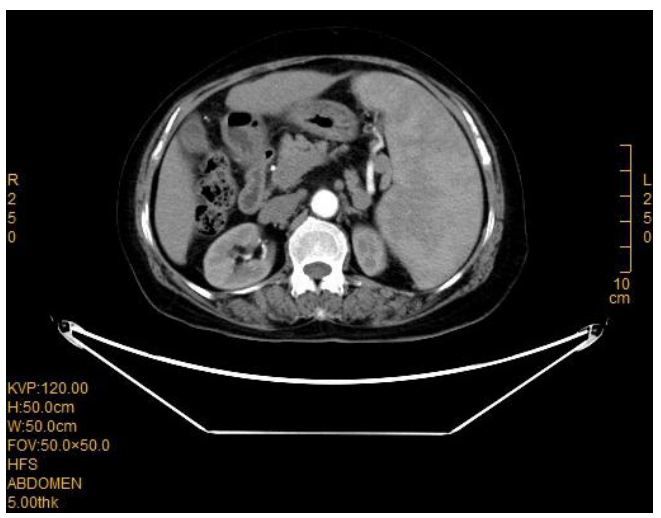

Figure 2 Abdominal CT scans: small round low-density shadows in the spleen and liver; intrahepatic bile ducts, splenic varices and portal veins were wide; splenomegaly. treated because of no apparent aches. Half a month ago, the patient suffered from anorexia and palpitations, shortness of breath, dizziness, and fatigue during physical activities. The patient did not feel chest pain, hematemesis, melena, abdominal pain, abdominal distension, urinary urgency, frequent urination, or painful urination. After accepting relevant examinations, she was diagnosed with splenic hyperfunction and thrombocytopenia and was hospitalized. The patient had no significant weight loss owing to the illness. The patient had a history of Sjogren's syndrome and hypertension but was not under regular treatment. She had no history of infectious diseases, diabetes, heart disease, drug and food allergies, and surgery or blood transfusion. The clinical examination of the patient did not reveal obvious abnormalities, excluding splenomegaly more than 3 $\mathrm{cm}$ below the costal arch. The auxiliary examination was as follows.

Abdominal ultrasound (Figure 1): The size and shape of the liver were normal and the capsule was intact, while the light spots were enlarged and the distribution was not uniform. No abnormalities were found in the gallbladder and pancreas. The size of the spleen was about $163 \times 55$ $\mathrm{mm}^{2}$, with a clear outline and uniform internal echo.

Abdominal computed tomography (CT) scans (Figure 2): Intrahepatic bile ducts in the right lobe of the liver showed mild dilatation, while small round low density shadows were found in the left lobe without enhancement. The spleen was enlarged and had many small, round, low-density shadows. Splenic varices and portal veins were slightly wide.

Gastroscopy: Varices were found in the lower part of the esophagus and the fundus of the stomach.

Echocardiography and chest x-ray film: No obvious abnormalities were seen.

Laboratory inspection: complete blood count (CBC): As illustrated in Table 1, the results of CBC showed that the platelet count decreased continuously until May 21 .

Blood biochemical examination: No obvious abnormalities were observed, excluding aspartate aminotransferase and

Table 1 The results of CBC

\begin{tabular}{lcccc}
\hline Date & WBC $\left(10^{9} / \mathrm{L}\right)$ & $\mathrm{RBC}\left(10^{12} / \mathrm{L}\right)$ & $\mathrm{Hb}(\mathrm{g} / \mathrm{L})$ & 84 \\
\hline $2019-05-11$ & 2.99 & 4.47 & 95 & 84 \\
$2019-05-16$ & 5.28 & 4.99 & 98 & 30 \\
$2019-05-20$ & 3.08 & 5.20 & 88 & 70 \\
$2019-05-21$ & 3.60 & 4.76 & 88 & 70 \\
\hline
\end{tabular}

CBC, complete blood count; WBC, white blood cell; RBC, red blood cell; Hb, Haemoglobin; PLT, platelet count. 
glutamyltransferase levels of 49 and $162 \mathrm{U} / \mathrm{L}$, respectively.

Immunology: HBsAg, HAV-IgM, and HEV-IgM were negative.

Peripheral blood smear (Figure 3): Platelet aggregation was detected.

\section{Diagnosis and treatment}

The diagnosis of splenomegaly and hypersplenism was confirmed according to the symptoms, signs, and the results of the auxiliary examination. After admission, the patients received a subcutaneous injection of recombinant human granulocyte colony-stimulating factor along with oral administration of butyl alcohol and leucogen to stimulate bone marrow hematopoiesis, and accepted tiopronin to improve her liver function. She had a history of Sjogren's

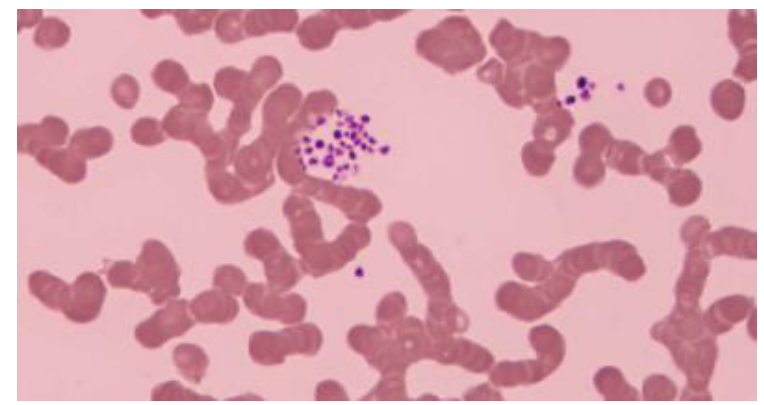

Figure 3 Platelet aggregation showed in Peripheral blood smear by Wright's-Giemsa staining $(\times 1,000)$.

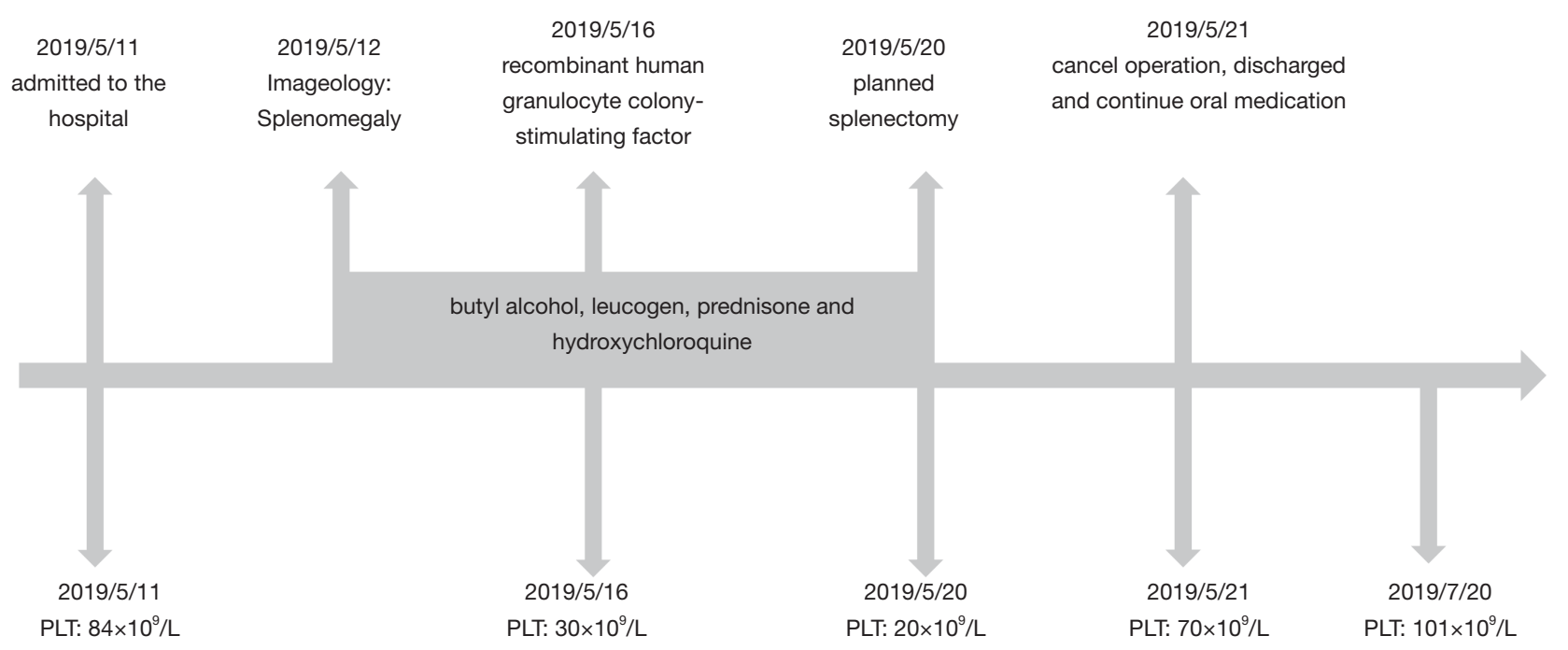

syndrome and was treated with prednisone $10 \mathrm{mg}$ tid and hydroxychloroquine 0.2 bid. On May 16, CBC revealed a drop in her platelet count to $30 \times 10^{9} / \mathrm{L}$. Considering the severity of hypersplenism, the clinicians planned splenectomy for the patient on May 20. Platelet aggregation was detected on the peripheral blood smear test.

Before the surgery, the doctor reexamined the blood routine. The results showed a platelet count of $20 \times 10^{9} / \mathrm{L}$ and platelet aggregation. The laboratory physician contacted the clinician and informed that the patient might be having EDTA-PTCP. The platelet count was $70 \times 10^{9} / \mathrm{L}$ on immediate detection after blood collection. Besides hypersplenism, the patient also had pseudothrombocytopenia. According to the actual platelet count, the patient did not require surgical treatment. The doctor discharged her and advised oral medication and periodic check-ups. The patient's appetite was improved without any adverse and unanticipated event during the follow-up. It was a great relief when her platelet count increased to $101 \times 10^{9} / \mathrm{L}$ on July 20 (Figure 4).

Written informed consent was obtained from the patients for publication of this manuscript and any accompanying images.

\section{Discussion}

The empirical diagnostic method for hypersplenism is B-ultrasound combined with blood routine test, but the accurate diagnosis depends on positron emission tomography-CT (PET-CT) or pathological biopsy. The 
clinical manifestations of hypersplenism are splenomegaly, thrombocytopenia, leukopenia, and anemia (4). Hypersplenism occurs after splenomegaly and can increase the retention and destruction of blood cells in the spleen, leading to thrombocytopenia. Hypersplenism is categorized into secondary and primary hypersplenism. Secondary hypersplenism is more common; it occurs as a result of portal hypertension, infectious inflammation, bone marrow hyperplasia, chronic hemolysis, and so on (5). According to the abdominal CT and B-mode ultrasonography, hypersplenism in the present case might be due to portal hypertension caused by cirrhosis of the liver. The medical history and the results of auxiliary examinations indicated that the cirrhosis was caused by Sjogren's syndrome. Therefore, besides stimulating bone marrow hematopoiesis to elevate platelets, the treatment of primary diseases using glucocorticoids and immunosuppressive agents should not be neglected. Because of the continuous decrease in the platelet count, the doctors in the present case believed that the conservative treatment of internal medicine was poor. So, they planned partial splenectomy for the patient.

Before the surgery, the clinician recommended to get a blood routine examination done to evaluate the condition. The laboratory physician accepted the suggestion and suspected that "platelet aggregation" was labeled in the inspection report, but the clinician still insisted on the blood routine test. Therefore, the physician communicated with the clinician. As a result of the communication, it was clear that the clinician did not understand that "platelet aggregation" might be a false reduction of the platelet count. This clarity in understanding the case helped avoid removal of the spleen, which had not reached the standard of splenectomy. The incidence of EDTA-PTCP was $0.09-0.21 \%$ (6). One of the reasons was that EDTA produced immune-mediated cold anti-platelet autoantibody, which was related to some latent antigen on the surface of the platelets. This can be corrected in several ways (7-9): blood sample collected with different anticoagulants for reexamination and smear microscopy; instrument dilution method, in which the examiner collects the blood, puts it in the dilution solution, and performs the test immediately; manual counting; without changing the anticoagulant, amikacin added to the blood for reexamination.

This is a rare and complex case of thrombocytopenia caused by two factors. In addition, the clinician lacks comprehensive understanding of the laboratory terms. It almost leads to misdiagnosis and mistreatment of the patient, which is the limitation of this case. Fortunately, the effective communication in the later stage guides the correct treatment of the patient. The present case study suggested the following: Firstly, medical staff should always maintain a rigorous work style and a high sense of responsibility. Disease diagnosis follows the basic principle of monism, but few cases cannot be excluded. For the case presented in this study, thrombocytopenia was due to hypersplenism and the use of EDTA-PTCP. However, the clinicians misdiagnosed the patient's thrombocytopenia as entirely due to hypersplenism, and planned splenectomy for the patient. Secondly, clinicians should fully understand the clinical significance of laboratory examination and commonly used laboratory terms. Laboratory inspectors should not rely excessively on automated instruments. They should do a good job of quality control in the three links of pre-analysis, mid-analysis, and post-analysis, and establish a sound review process for abnormal results. When the result is abnormal, a combination of histogram, scatter plot, and alarm tips should be used. If the abnormal result meets the reexamination standard, manual counting and smear microscopy should be conducted to obtain accurate data. The audit should not be easily passed, and an untrue report should not be issued based on meeting the quality control requirements. Finally, active, timely, and effective communication is very important. Laboratory physicians should establish effective communication with clinicians through various ways, such as regularly introducing the clinical significance of new projects to clinicians, guiding specimen collection, and explaining professional terms. If an abnormal and suspicious result is encountered, the laboratory physician should communicate with the clinician in time and find out the cause with the clinician to ensure the accuracy and reliability of the result. Effective communication between laboratory physicians and clinicians can avoid missed diagnosis and misdiagnosis of diseases.

\section{Acknowledgments}

Funding: This work was supported by Project of Jiangsu Provincial Health Commission (H2018106).

\section{Footnote}

Conflicts of Interest: All authors have completed the ICMJE uniform disclosure form (available at http://dx.doi. org/10.21037/apm-19-295). The authors have no conflicts of interest to declare. 
Ethical Statement: The authors are accountable for all aspects of the work in ensuring that questions related to the accuracy or integrity of any part of the work are appropriately investigated and resolved. All procedures performed in studies involving human participants were in accordance with the ethical standards of the institutional and/or national research committee(s) and with the Helsinki Declaration (as revised in 2013).Written informed consent was obtained from the patients for publication of this manuscript and any accompanying images.

Open Access Statement: This is an Open Access article distributed in accordance with the Creative Commons Attribution-NonCommercial-NoDerivs 4.0 International License (CC BY-NC-ND 4.0), which permits the noncommercial replication and distribution of the article with the strict proviso that no changes or edits are made and the original work is properly cited (including links to both the formal publication through the relevant DOI and the license). See: https://creativecommons.org/licenses/by-nc-nd/4.0/.

\section{References}

1. Britt RS, Pearson JC, Mahoney MV, et al. CeftarolineInduced Thrombocytopenia: A Case Report. Ann Pharmacother 2019;53:762-3.

Cite this article as: Zhu Y, Pan Y, Hou PF, Zhu LJ. Ineffective communication equals no communication: a case report of splenic hyperfunction combined with pseudothrombocytopenia. Ann Palliat Med 2020;9(5):3597-3601. doi: 10.21037/apm-19-295
2. Fujikawa K, Koga T, Honda T, et al. Serial analysis of cytokine and chemokine profiles and viral load in severe fever with thrombocytopenia syndrome: Case report and review of literature. Medicine (Baltimore) 2019,98:e17571.

3. Riley DS, Barber MS, Kienle GS, et al. CARE guidelines for case reports: explanation and elaboration document. J Clin Epidemiol 2017;89:218-35.

4. Gu JJ, He XH, Li WT, et al. Safety and efficacy of splenic artery coil embolization for hypersplenism in liver cirrhosis. Acta Radiol 2012;53:862-7.

5. Wu Y. Current status and new progress in surgical treatment of liver cirrhosis with hypersplenism. China Practical Medical 2016,11:277-9.

6. Kurata Y, Hayashi S, Jouzaki K, et al. Four cases of pseudothrombocytopenia due to platelet cold agglutinins. Rinsho Ketsueki 2006;47:781-6 .

7. Shi J, Xiao T, Lu HM, et al. EDTA-dependent thrombocytopenia: a case report. Lab Med Clin 2018;15:3638-9.

8. Huang XH, Lin J, Hao QF, et al. Analysis and treatment of EDTA-dependent pseudothrombocytopenia. Experimental and Laboratory Medicine 2018;36:46-8.

9. Zhou X, Wu X, Deng W, et al. Amikacin can be added to blood to reduce the fall in platelet count. Am J Clin Pathol 2011;136:646-52. 